Mr. Hill laid stress on the importance of proceeding at once to herniotomy in cases presenting symptoms of strangulation, when the ordinary accessory means have been tried unsuccessfully; and added that, in cases where the tumour is of an obscure nature, it is far better to explore by incisions than to leave the case to chance, the more so as herniotomy without the sac is a simple operation. The case in question showed how necessary it is carefully to examine every step in these operations, since it seldom happens that two cases are exactly alike, and some important and dangerous anatomical deviation or abnormality may arise in the practice of the most experienced surgeon, if any set rule be adopted as to the modus operandi.

\section{EVELINA HOSPITAL FOR SICK CHILDREN.}

\section{A CASE OF EMPYEMA, TREATED BY PARACENTESIS AND} SUBAQUEOUS DRAINAGE.

(Under the care of Dr. W. S. Playfarr.)

For the notes of this case we are indebted to $\mathrm{Mr}$. Clement Godson, lately medical registrar to the hospital.

Thirty-eight days before admission the patient had caught cold during the night, and awoke with pain in the side. On admission, the left chest measured three-quarters of an inch more than the right, was universally dull, and presented no intercostal depressions; the heart beat about half an inch below the right nipple; the temperature was very variable. All the other physical signs combined to indicate that the pleura contained fluid.

A few days after admission it was found advisable to velieve the chest by paracentesis, and about a pint and a half of purulent fluid was evacuated, to the manifest relief of the patient. In eleven days more the operation became again urgently necessary, and on this occasion the fluid was conveyed by means of a piece of india-rubber tubing, which entered the cavity of the pleura and was fixed to the chest by means of wire and strapping, into a bottle partly filled with water, through the cork of which it passed; the extremity of the tube fell below the level of the water. A pint and three-quarters came away on this occasion. During each of the three succeeding days about two ounces of pus were discharged into the bottle, for some more days about an ounce, then for a few days half an ounce, and so on, until on the thirty-second day the discharge became so slight that the apparatus was removed, and a small strip of lint was introduced into the wound. In a few days more this also was discontinued, and the fistula was allowed to close. On the forty-second day the wound had closed and the child was in good health. In a few days more she was sent in to the country. When she was discharged there was some slight contraction of the chest-wall below the clavicle, but the heart had resumed its natural position, and normal resonance and good respiratory murmur had been everywhere established.

\section{THE}

\section{ADDRESS IN MEDICINE \\ DELIVERED BEFORE THE}

\section{ANNOAL MEETING OF THE BRITISH MEDICAC} ASSOCIATION, AT EDINBURGH,

\section{By GEORGE JOHNSON, M.D., F.R.C.P.,}

PROFESSOR OF MIEDICINY TN KTYG'S COLKEGE, PHYYSTCIA TO KING'S COLLEGE HOSPITAL, BTC.

\section{NATURE AND ART IN THE CURE OF DISEASE}

MaNY of those whom I have now the honour to address will remember how great an interest was excited when, about fourteen years ago, the late Sir John Forbes published his book on "Nature and Art in the Treatment of Disease." The author of that little volume, in clear and vigorous language, with pitiless logic, characteristic truthfulness, and fearless candour, pointed out the evils resulting from what he calls the over-active perturbative treatment and the mischievous polypharmacy which were then prevalent.
Sir John Forbes lamented that in his day a purely expectant treatment of disease was rarely practised, except under other colours and under other names; and he referred to the results of homœopathic treatment, which he, with reason, looked upon as simply inert, in proof of the proposition that " the power of Nature to cure disease is infinitely greater than is generally believed by the great body of medical practitioners and by the public. So great, indeed, is this power," he goes on to remark, "and so universally operative, that it is a simple statement of facts to say that, of all diseases that are curable and cured, the vast majority are cured by Nature independently of art ; and of the number of diseases that, according to our present mode of viewing things, may be fairly said to be curable by art, the far larger proportion may be justly set down as cured by Nature and art conjointly." He elsewhere remarks that cases recorded by young practitioners in the medical journals afford additional evidence of a yet stronger kind, by showing, not simply the power of Nature to overcome natural disease, but to overcome this and the artificial disease superadded by what he very unpolitely calls " the energetic ignorance of the practitioner."

To the same effect, many years before, Lord Byron, when balf-dead of a fever in Greece, wrote what he intended to be his own epitaph :-

\section{"Youth, Nature, and relenting Jove, To keep my lamp in strongly strove; But Romanelli was so stout, He beat all three, and blew it out."}

After all, however, he recovered on that occasion, and he adds that " Nature and Jove, being piqued at my doubts, did, in fact, at last beat Romanelli ; and here I am, well, but weakly."

Since the publication of Sir John Forbes's book, and partly, no doubt, in consequence of that publication, our views as to disease and its treatment have undergone a very creat change. A purely expectant treatment is now as common as then it was rare. It is now fashionable and orthodox to trust to the curative powers of Nature, and to doubt the therapeutic power of art. The pendulum has swung from one extreme to the other. At that time it was said, " that according to the vulgar notion, the function of the physician consists in little else than the prescription or administration of drugs, and the function of the patient in little else than swallowing them." Now, on the contrary, that which was once said satirically, has come to be an almost accepted rule of practice-namely, that " the chief business of the physician is to amuse the patient while Nature performs the cure."

Now it is a very noteworthy fact that, simultaneously and side by side with this firm belief in the almost allsufficiency of Nature and the impotence of art, there notoriously exists an extreme unwillingness to admit that any phenomena of disease $c$ in rightly be considered as having a conservative or curative tendency. The writer of an article "On the Pathology of the Microscopic Arteries," in the April number of the British and F'oreign MedicoChirurgical Review, had good reason for the statement which he there makes, that to speak of pathological processes as curative efforts of Nature, " is a mode of looking at the phenomena of disease always suspiciously cross-examined at the present day." This statement, however, suggests the inquiry whether a suspicious cross-examination is not uncalled for and out of place when the object is to arrive at truth, and not merely to gain a verdict. For the discovery and appreciation of scientific truth, the judicial impartiality of the bench is certainly more effective than the zealous and sometimes not over-scrupulous partisanship of the bar.

Surely there is is priori good reason to believe that in the curative processes of Nature there is an orderly method of procedure, of which, by a diligent search, we may gain some useful knowledge. That marvellous reparative power which cnres a fever or an inflamed lung as thoroughly as it heals a wound or mends a broken bone, must certainly work after a definite plan in each class of cases. The characteristio manner in which, during the progress of acute febrile and inflammatory diseases, the temperature rises, fluctuates, 
and finally falls, within a period which, for each disease, has a more or less exact limit, is one amongst other indications of such a definite plan and order. And as conservative surgery has been much advanced by a careful study of Nature's method of healing wounds and repairing injuries, so it can scarcely be doubted that a thorough analysis of the more obscure phenomena of internal diseases, and an investigation of their physiological sequence and relationship, may afford us great assistance in our endeavour to conduct our patients safely through the storm of a dangerous sickness. Indeed, I hope to carry you with me in my attempt to show that much knowledge of this kind is already available and ready for daily use.

We are all too apt to forget that disease is a natural, although an abnormal condition of the body; that pathology is, in fact, a department of physiology, and that the phenomena of disease result from the action of the normal structures and forces only modified by morbid conditions. To take an extreme case, the time, perhaps, is not far distant when we may be able to give a physiological interpretation of even that destructive deviation from the normal processes of nutrition which results in the disease recognised as cancer. Meanwhile much aid to soientific pathology and practical medicine is to be expected from the improved teaching of physiology, to which so much attention is now being given. Unquestionably there is a great call for improved and extended physiological training, for there are manifest signs of a lamentable ignorance of the subject, not only amongst otherwise highly educated laymen, but amongst medical writers who publicly discuss physiological questions, and who therefore subject themselves to public criticism. You will not have forgotten that a very influential newspaper lately endeavoured to excite the sympathy of its readers and to bring discredit upon an eminent professor, by commenting on the supposed sufferings of a decapitated frog, whose automatic movements were assumed to be the result of volition, and to be indicative of pain. Then, if anyone desires to test the physiological acquirements of certain writers on pathology, let him study, as I have done, the copious literature of cholera. He will find, amongst other anomalies, that one writer of great eminence considers that he has sufficiently explained the collapse of cholera when he pronounces it to be the result of "paralysis of the sympathetic nerve." Another writer on cholera, having satisfied himself by experiment that the disease is communicated by the poisonous discharges from the alimentary canal, maintains that the poison does not become absorbed so as to enter the circulation, but that it has purely local action upon the epithelial lining of the bowel.

Now, the rapid absorption and diffusion of all known poisons as an essential condition of their operation is one of the best established of physiological doctrines. The interesting observations of Dr. Bence Jones demonstrated, not only the rapidity of absorption from the alimentary canal, but also the rapid diffusion of the absorbed materials out of the vessels even into the non-vascular tissues. These physiological doctrines, as well as the numerous facts which go to prove the absorption of the cholera poison, are ignored by the author of the theory to which I have referred; and if we accept his teaching, we are required to believe that in the case of the cholera poison a general law of physiology is suspended.

I propose now to refer to some very obvious and wellknown phenomena as examples of morbid processes having a conservative or curative tendency. Let us take, for instance, the case of a patient who is jaundiced in consequence of mechanical obstruction of the duct by a gall-stone. His urine is charged with bile, the result of a vicarious excretion of bile products by the kidneys. It will scarcely be denied that the elimination of bile through the urinary channels is, on the whole, a wholesome conservative process, tending, as it does, to free the blood from impurities, the retention of which might be injurious, and even fatal. Yet this vicarious excretion is not effected without some functional disturbance, and even temporary structural change in the kidney. An examination of bilious urine often discovers in it desquamated renal epithelium, tube casts, and sometimes albumen-pathological conditions which quickly pass away when the bile, resuming its natural course, ceases to be excreted by the kidneys.

Again, in a case of diabetes there is an abundant secretion of saccharine urine. Now, whatever may be the primary seat and essential cause of diabetes, it is certain that the free elimination of the excess of sugar by the kidneys is ssentially a beneficial and life-preserving process. Yet this continued secretion of sugar not unfrequently induces structural changes which ultimately lead to a fatal result. The persistent elimination of sugar by the kidneys has the effect of changing the structure and the vital properties of their secreting cells, so that, in adapting themselves, as it were, for the secretion of sugar, they gradually become unfitted for the discharge of their own proper function-namely, the secretion of urine. The renal gland-cells at length become opaque, and are found to contain a large amount of finely granular material with oily particles. The circulation through the kidney becomes impeded, the gland is congested, its secretion is albuminous, and suppression of urine is the immediate cause of death in a large proportion of cases of diabetes.

I believe that the familiar facts to which I have here briefly referred afford a good illustration of a principle which admits of a very extensive application to the interpretation of morbid phenomena. A large number of dis. eases are caused by, or at any rate associated with, some form of blood-poisoning. The morbid poisons and their products are eliminated through various excretory channels, more especially through the kidneys, the liver, the skin, the lungs, and the mucous membrane of the alimentary canal. In many instances the elimination of the morbid poison causes much functional disturbance and structural change in the excretory channels through which the poison escapes and these structural changes constitute the most striking outward signs and diagnostic marks of the diseases with which they are associated. Thus we have the cutaneous eruptions of the acute exanthemata, the gastro-intestinal symptoms of cholera, the bowel disease of dysentery and enteric fever, the hepatic disease which results from excess of alcohol or from the influence of malarious poisons, the renal disease, which may also be caused by an excess of alcohol, by the poison of scarlet fever, and by various othermorbid poisons which I need not now stop to particularise.

Here let me say that I am perfectly well aware that by a certain class of pathologists the doctrine of the elimination of morbid poisons is ridiculed as a vestige of a bygone, unenlightened age, well enough adapted to please the faney of old women and ill-educated laymen, but quite unworthy the acceptance of scientific physicians. One of this very advanced school lately intimated to me privately that, as a supporter of this exploded notion, he looked upon me as the priest of a decaying faith. Now it is a remarkable fact that the opponents of the doctrine in question not unfrequently render it ridiculous by misrepresenting it; the misrepresentation being, of course, unintentional and apparently an unconscious result of confusion of ideas in the minds of the critics. Thus, quite recently in one of the medical journals, an eminent provincial physician asks what we mean by the term "disease"; and he replies by sasing: "In old times people used to think that a disease was some actual entity or thing which had got into the body in some way and was there lying hidden and secreted, and was to be cast out. We know that there are a few instances of this kind of thing; for example, we know that some disorders depend upon the presence of a tapeworm in the intestines, and that to cure them we must hunt the worm out of the body. This idea, which we know to be true only in a few specific instances, was at one time general. The germ of this idea still runs through a great many of our conceptions of the nature of disease. This is more particularly the case in the pathological conceptions of illeducated laymen; but many traces of it are still to be found amongst ourselves also."

I venture to suggest that the writer of the passage which I have here quoted confounds, as others equally eminent have done before, the idea of disease with that of a morbitic cause. If anyone maintains, or ever did maintain, that disease is an entity, having an existence apart from the living body, I am not here to defend so manifest an absurdity. I apprehend that what we all understand by disease is an abnormal condition of the living body. The canses of disease are almost infinitely various. Some disea ses have their origin in purely mental, emotional, or nervous influences, but the cause of many diseases is as certainly, if not as obviously, a separate entity, as the ovum of a tapeworm. For instance, the disease which we call lead-colic is the 
pain with constipation experienced by a man who has been poisoned by lead. The colic, of course, can have no existence apart from the sufferer, but the metallic poison which canses the colic was introduced from without, and may be again ejected from the system. We know that small-pox and scarlet fever can exist only as phenomena of living bodies, but the cause of each of these diseases is a material and portable poison, which, being thrown off from the bodies of the sick, conveys the disease to the healthy. The theory of contagion as generally accepted, and the practice of disinfection as commonly adopted, both imply a belief in the elimination of morbid poisons. This doctrine of the elimination of morbid poisons is of course quite consistent with a belief that in part they may be decomposed and destroyed within the system, although we can have no proof of the actual occurrence of this destructive process.

I almost feel that I owe you an apology for occupying your time by insisting upon such obvious, elementary truths. It is strange that at a time when so much discussion is going on as to the nature of morbid poisons, when germs and dust and fungi, their nature and origin, and operation upon the living body, and the best mode of preventing or neutralising their effects, form the subjects of fierce controversy, there should occasionally be heard a confused utterance which seems to imply that the whole controversy is utterly unpractical and unmeaning; that while a tapeworm is an undoubted fact, morbid poisons are the phantom prodncts of a disordered brain. As an illustration of this kind of unreasonable scepticism, I may remark that a recent writer on cholera in India, Mr. Macnamara, denies the existence of a blood-poison in that disease, because Dr. Thudichum failed to obtain chemical evidence of such a poison. As if Dr. Thudichum had chemial proof of the existence of any of the morbid poisons of whose reality and destructive activity the evidence, other than chemical, is only too conclusive.

And now to return to the subject of curative efforts of nature, and the conservative tendency of certain morbid processes. There are few diseases which afford more striking illustrations of the principle in question than the various forms of Bright's disease, when traced through all their stages, from their orioin to their termination. Excluding from consideration those cases of albuminuria which are caused by a mechanical impediment to the circulation, the result, usually, of cardiac or pulmonary disease, it may be held as a doctrine generally true that the primary cause of Bright's disease in all its forms is a morbid condition of blood, and that the structural changes which the kidney undergoes are the result of a conservative effort to excrete noxious materials from the circulation. In cases of transient blood-poisoning, scarlet fever for example, the structural changes in the kidney and the functional disturbance may be only temporary, and the recovery complete. But in other instances, as, for example, cases of chronic alcoholism-a very frequent exciting cause of Bright's disease,-the continued passage of noxious products through the secreting structures of the kidney gradually destroys the tissues, and the gland either wastes and contracts, or becomes so structurally changed as to be unsuited for the discharge of its functions. There is then a secondary blood contamination, a result of retained urinary excreta, and this uræmic condition may, in a variety of ways, bring about a fatal result. In illustration of our present subject, it may be observed that when the renal disease has reached an advanced stage, life is often prolonged for a time by the vicarious elimination of urinary excreta through the mucous membrane of the alimentary canal. It is probable, too, that the dropsical symptoms which often complicate renal disease are the result of a conservative effort to free the blood from an excess of water, whose retention within the vessels would be more detrimental than its accumulation in the subcutaneous tissue and the serous cavities. .....

[In further illustration of conservative morkid processes, Dr. Johnson directed attention to certain changes of alnost constant occurrence which are found in the heart, and in the minute arteries, after death from chronic Bright's disdisease; and dilated upon the doctrine. He then proceeded.]

In these days of specialism, when the tendency is to dissever the various organs of the body, and to forget their physiological relationship to each other, it is interesting and instructive to find that the deterioration of the blood which results from the degeneration of one organ-the kidney-may induce hypertrophy not only of the walls of the heart, but also of the walls of the minute arteries in every tissue throughout the system. A good illustration this of the very ancient doctrine that, "if one member suffer, all the members suffer with it."

Time will not permit me to comment upon the various symptoms of Bright's disease which receive their explanation from the anatomical changes which I have described. There is, however, one phenomenon to which I must make a passing reference. A full, hard, resisting radial pulse, with increased arterial tension, as indicated by the sphygmograph, is commonly observed in cases of chronic Bright's disease. The explanation is obvious, by reference to the two antagonistic forces, that of the strong left ventricle, and that of the equally strong resisting arterioles. So that, when the history of a case and the chemical and microscopical characters of the urine are insufficient to determine the question whether the renal disease is chronic or of recent origin, the physical signs of hypertrophy of the left ventricle, with increased arterial tension, afford important confirmatory evidence.

[Dr. Johnson next defended the teleological argument as applied to the structure and functions of the living body, whether in health or in disease. He then continued.]

During the time which remains to me to-day, my purpose will be to show that, as prastitioners of medicine, we have something more to do than to watch the phenomena of disease as passive spectators, and that in our endeavour to prevent, to mitigate, and to cure disease, we have a better guide than mere empiricism.

Obviously, one of the most essential conditions for the successful treatment of disease is an exact diagnosis. If diseases essentially different are confounded together, it is impossible to arrive at any trustworthy conclusion as to the effect of particular remedies or plans of treatment. Nor does it suffice for successful treatment that a disease be correctly named and referred to its right place in a nosological system. I need only refer to the oroup of cases which are designated epilepsy to illustrate this proposition. There is reason to believe that the immediate cause of an epileptic convulsion is in all cases identical-namely, a sudden and extreme anæmia of the brain; but the remote and exciting causes of this cerebral anæmia are numerous and very diverse. It may be a result of failure of the general circulation, consequent upon profuse and rapid hæmorrhage, or a plug of fibrine (embolism or thrombosis) in the pulmonary artery; or the pulmonary, and consequently the systemic, circulation may be rapidly arrested by one of the various forms of apncea, or by the accidental admission of atmospheric air into the veins during the performance of a surgical operation at the root of the neck or in the axilla. But in most cases of epileptic convulsion, the arrest of the circulation and the cerebral anæmia are probably due to a sudden and extreme contraction of the minute intracranial arteries. The exciting causes of this arterial contraction are very various. It may be the result of a purely nervous or reflex influence acting, through the vaso-motor nerves, upon the cerebral vessels. Examples of this form of epileptic convulsion are afforded by cases in which the determining cause is emotional excitement or mental anxiety, syphilitic or tuberculous inflammation of the brain or its membranes, painful dentition, the irritation of intestinal worms. In another class of cases blood-poisoning is the immediate cause of the arterial contraction. To this group belong cases of uræmic convulsions-convulsions the result of alcoholism, and the convulsions which sometimes occur during the initiatory fever of the acute exanthemata. If the treatment of epileptic convulsions is to be successful, or even harmless, we must do something more than give the disease a name, and prescribe bromide of potassium, valuable as that remedy is in many cases of this terrible disease.

We are sometimes told that our chief duty as practitioners is to treat symptoms, and to leave pathological theories to those who have nothing better to occupy their time and attention. A patient consults us for aphonia; shall we presribe for this symptom without looking into the larynx to ascertain its cause?

Again, when it is said that we are to treat symptoms, is it meant that we are to make it a rule of practice to endeavour to repress all the symptoms of disease without re- 
ference to their origin, their physiological significance, or their probable conservative tendency? Such a rule would lead to the repression of the eruption of scarlet fever and small-pox by external cold, to the abrupt arrest of choleraic discharges by opinm, and to the weakening of a strongly pulsating hypertrophied heart associated with valvular disease by low diet and depletion-modes of treatment condemned alike by pathological science and by the results of experience.

In estimating the results of treatment, the ultimate appeal must obviously be to experience. Experience, however, has notoriously proved, in many instances, a fallacious guide : experientia fallax is a maxim which we shall all do well to keep continually in mind.

But of all the delusive guides in practice a false theory of disease is the most to be dreaded. It was an erroneous theory that suggested the antiphlogistic treatment of delirium tremens and the narcotic and alcoholic treatment of cholera; and theory alone prevented the more speedy discovery and rectification of these practical errors.

A knowledge of the causes of disease, and of their modus operandi in the production of morbid phenomena, may be made practically useful in many and various ways. The evidence obtained within the last few years of the manner in which cholera and enteric fever spread through the contamination of the air and drinking-water by the intestinal discharges has greatly increased our power of preventing these destructive diseases. The ascertained influence of comparative dryness of soil and of atmosphere, resulting from improved drainage, in diminishing the mortality from phthisis in various parts of America and in this country, forms another important contribution to practical and preventive medicine.

There is a numerous and important class of cases in which suppressed action of the skin by cold or by some unknown atmospheric influence is the exciting cause of disease in internal organs : for example, cases of acute renal dropsy, whether occurring during the progress of scarlet fever or unconnected with that disease; cases of catarrh and bronchitis; cases of acute pneumonia ; and some cases of acute rheumatism. Now there is a principle of practice common to all these cases, which is this-that if at the very com. mencement of the disease a free action of the skin can be promoted, the malady may often be cut short, or, if not entirely and promptly arrested, it will be greatly mitigated. It is notorious that an ordinary catarrh may almost certainly be cured by free diaphoresis at the very commencement of the attack. I have myself gone into a hot-air bath suffering from headache, pain in the limbs, and other indications of a severe incipient catarrh, which, if allowed to run its course, would probably continue for a period of from one to two or three weeks; and in the course of half an hour the symptoms have been entirely removed by the action of the batb. The same treatment by hot-air or blanket baths is certainly very efficacious in the early stages of acute renal dropsy, more especially when cold has been the exciting cause of the disease. The object of the diaphoretic treatment in these cases is not, as some writers appear to imagine, to sweat urine through the skin, but, by diverting a large amount of blood to the surface, to lessen the congestion of the kidney - as dry cupping over the loins does in a less degree, - and thus to increase the secretion of urine. The objection which has been made to this sweating practice-that it tends to waste a portion of the water which is required to wash morbid products from the uriniferous tubes-affords a good illustration of the insufficiency of mere physics to explain physiological phenomena.

[In connexion with this diaphoretic practice, Dr. Johnson referred to the treatment of catarrhal pneumonia, and stated that, by treatment promptly applied during the febrile prepneumonic stage, the amount of subsequent exudation may be greatly lessened. The treatment suggested by theory and approved by experience consists in the prompt restoration of the suppressed cutaneous secretion, by hot air or water, or wet sheet and blanket baths, an emetic of antimony or ipecacuanha when there is nausea without vomiting, and a calomel and colocynth pill, followed by a saline purgative.]

It remains for me now to sum up in a few sentences the main points which I have had the privilege to bring before you.

I have suggested that a belief in the power of Nature to cure all curable diseases is inconsistent with a disbelief in the existence of morbid processes having a conservative or curative tendency. I have indicated various pathological phenomena, the conservative tendency of which appears to me indisputable; and I have endeavoured to show that, by a careful study of the functional and structural changes which result from disease, we may obtain most valuable indications for treatment-learning thereby both to do that which may aid Nature, and to avoid such means as may tend to thwart and hinder the natural curative processes.

Again, $I$ have intimated that it is difficult, and, as it seems to me, impossible, to reconcile a disbelief in the elimination of morbid poisons with a belief in the spread of disease by contagion. Confirmatory evidence as to the elimination of morbid poisons is afforded by the disastrous results of repressive methods of treatment. To take all possible precautions to exclude the cholera poison from the system, and then, when once it has gained an entrance, to endeavour to retain it there by opiates and astringents, are practical modes of procedure ntterly inconsistent with each other; unless, indeed, the object of this repressive treatment be to sacrifice the individual for the public good - to prevent the patient, at the peril of his own life, from scattering the seeds of disease and death amongst the community.

I believe that the success of our attempts to cure and to prevent disease depends mainly upon an exact diagnosis and discrimination of the various forms and shades and stages of disease; upon a correct interpretation of pathological processes and symptoms; a careful avoidance of erroneous and misleading theories; and, lastly, upon a prompt recognition of the exciting causes of disease, some of which may be avoided, some removed, while the influence of others may be in a greater or less degree counteracted by the timely employment of suitable means.

\section{BRITISH ASSOCIATION FOR ADVANCEMENT OF SCIENCE.}

Meeting at Edinburgh, AUg. 2No to 9th inchustre.

AFTER an interval of many years, the Association was again hospitably received in the modern Athens; and, what is a rare thing at such gatherings, all the various Sections were accommodated in that noble building, the University, which greatly added to the comfort and convenience of the members. This occurred also in 1859 at Aberdeen, when Marischal College, a fine modern building, was equally commodious. As might be expected in such a renowned school as that of Edinburgh, many subjects interesting to the physiologist and medical philosopher were brought forward, of which we shall endeavour to give a brief analysis.

The Section of Biology was subdivided into three departments - Anatomy and Physiology, Anthropology, and Zoology and Botany. Professor Allen Thomson, of Glasgow, delivered an address before the first, and Professor Turner, of Edinburgh, before the second. 'The latter gentleman stated that this was the first time in Scotland that an anthropological department had existed. In the course. of his remarks he asked the signification of the term "anthropology" among men of science. Mr. Wallace had defined it to be the science which contemplates man under all his varied aspects (as an animal and as a moral and intellectual being), in his relations to lower organisms, to his fellow-men, and to the universe. "It is obvious," Professor Turner remarked, "that a science thus defined is most comprehensive in its scope; that it embraces the nature and constitution of man, physically, psychically, and morally; the differences and resemblances between man and other organisms; his habits and language ; his history. past, present, and future." He then traced the history of this department, its struggle for existence, and the position it was likely to hold for the future.

Dr. B. W. Rrchakdos read the ninth of a series of reports "On the Pbysiological Action of Organic Chemical Compounds." The series was commenced at Newcastle in 1863. The substances described in the present report were 